\title{
Metamodeling to Ontology Conversion with Social Media Influence in Flood Management Domain
}

\author{
Muhammad Mahmud, Azman Yasin, Mazni Omar
}

\begin{abstract}
Disaster is an unpredictable event unknowingly to human being when and where it will happen. Nevertheless, early precautions can be made by initiating Disaster Management (DM) to reduce losses and destructions that may arise. Based on the impact seen from recent events globally, DM has been a focus area constantly developing and evolving. Leveraging emerging technologies such as social media can improve actionable intelligence in Situational Awareness aspect. Among other meteorological events, flood has been the most common natural disaster by far, cumulating in total $43 \%$ out of all disaster phenomenon from the last 20 years. However, in DM perspective, flood is still informally represented to enable information exchange and comprehensive flood management activities. To address both issues, this study will further extend existing Disaster Management Metamodel (DMM) with social media concepts and designs Flood Management by applying metamodeling process with UML class diagram since it has been well known by domain experts. Still, class diagram unable to cater applications that relies on processing content of information instead of visualization only. This limitation is overcome by transforming the metamodeling source into ontology format which elevates the expressiveness, meaning, and delivering more structured information among human or software agents. The reconstructed design of DMM successfully identifies new concepts generally and for flood domain. After running the mapping algorithm, the generated ontology is validated with Protégé to ensure the markups are persistence with the latter.
\end{abstract}

Index Terms: Flood Management Knowledge, Metamodel, Ontology, OWL, UML Class Diagram, Social Media.

\section{INTRODUCTION}

Disaster is an adverse event causing catastrophic impact globally on life, monetary, and infrastructures. With climate changing rapidly future natural disasters most likely will happen more frequent, without indicators, and difficult to forecast (Porto de Albuquerque et al., 2017).

Revised Manuscript Received on June 22, 2019.

Muhammad Mahmud, Human-Centered Computing Research Lab, School of Computing, Universiti Utara Malaysia (UUM), 06100 Sintok, Malaysia

Azman Yasin, Human-Centered Computing Research Lab, School of Computing, Universiti Utara Malaysia (UUM), 06100 Sintok, Malaysia

Mazni Omar, Human-Centered Computing Research Lab, School of Computing, Universiti Utara Malaysia (UUM), 06100 Sintok, Malaysia
These phenomena triggers immediate and long term socioeconomic effects throughout the world in a large scale (Jha, Barenstein, Phelps, Pittet, \& Sena, 2010). As seen in 2013 , disaster has affected monetary loses of nearly US $\$ 135$ billion and devastating 20500 casualties. Additionally, 44\% of these numbers, $49 \%$ of the casualties, and $37 \%$ of the monetary depletion were related to severe hydrological events (Munich, 2013). To reduce the impact caused by disaster events, an effective strategy is crucial to drive response and recovery phases. Reinventing the wheel in the sense of having an organized process to facilitate this activities can be addressed by initiating Disaster Management (DM). DM involves collaboration of problem solving activities, benchmarked with severe complexity in terms of various knowledge source spread over time, space, and human (Othman, Beydoun, \& Sugumaran, 2014). This area can be perceived as a unified process of designing, classifying, coordinating, and applying initiatives necessary for efficiently managing the affect with human (Deshmukh, Ravindra, Lewlyn L. R. Rodrigues, 2008).

Four main phases of DM are indicated as Mitigation, Preparedness, Response, and Recovery (Seneviratn, Chaminda Pathirage, Dilanthi Amaratunga, \& Richard Haigh, 2011). Mitigation or known as risk reduction phase consists of Structural and Non-Structural Mitigation steps adapted to prevent the disruptive aftermath of natural disasters (Atmanand, 2003; Bosher, Andrew Dainty, Patricia Carrillo, \& Glass, 2007; Lloyd-Jones, Kalra, Mulyawan, \& Theis, 2009; Moe, Gehbauer, Senitz, \& Mueller, 2007). Next phase Preparedness consults on activities and action taken prior in assuring credible response to the effect of disasters. This cover the release of advance warnings and short terms disaster clearance of civilians and infrastructures from endangered spot (Atmanand, 2003; Moe et al., 2007). Notice significant difference between Mitigation and Preparedness is mitigation took place in advance before the disaster take place while preparedness is instantly on the time the event being warned. Response phase comprise of help and aids at the moment of disaster occurrence to achieve life sustainability and essential need of hazard victims (Moe et al., 2007). The final phase which is Recovery focuses on reassembling from the disaster impact with the means of prolong longevity survival (Moe et al., 2007).

\section{Published By:}




\section{Metamodeling to Ontology Conversion with Social Media Influence in Flood Management Domain}

In general disasters are classified into nature and human caused (Slamet et al., 2018). Nature caused disaster consists of meteorology (flood, heat waves, rapid fire, hurricane, typhoon, thunder storm, and soil erosion) and geology type (natural earthquake, seismic activities, tsunami, and volcanic movement) while human or social caused disasters includes building structure, tunnel and bridge collapsed, hazardous chemicals, radiology garbage spill, nuclear, dam malfunction, and train accidents (Calton \& Masaru, 2013; Rahmat, Hagishima, Ikegaya, \& Tanimoto, 2016). Based on a joint report produced by United Nation (UN) for Centre for Research on the Epidemiology of Disasters and Disaster Risk Reduction (UNISDR) identifies the most common natural disasters is flood with 3062 record of occurrences cumulating $43 \%$ out of all disasters, $28 \%$ for storm, and $29 \%$ for others such as earthquake, extreme temperature, landslide, drought, wildfire, and volcanic activities between the year of 1995 to 2015 (Wallemacq, Guha-Sapir, McClean, CRED, \& UNISDR, 2015). From this statistics, it is critical to facilitate another form of disaster management which is flood to strategically control and oversee the provision of assistance before, during, and post events of hazard.

Information and communication plays a pivotal role during disaster response process. Communication is a vital element but often restricted as network facilities outage and overloaded hence urging improvement in DM planning (Underwood, 2010). Due to recent technological disruption especially social media and mobile have revolutionize the landscape of disaster management by allowing victims to share real time and specific information on happening events. Two main benefits from technology advancement in DM can be seen from data and communication oriented view point (Poblet, GarciaCuesta, Casanovas, García-cuesta, \& Casanovas, 2014). Data oriented approach empower additional data sharing such as before incident activity, incidents imminent real-time notice, and public reaction to emergency notification (Cameron, Power, Robinson, \& Yin, 2012). Seamless interaction between public and victims are enabled through communication oriented approach. Leveraging social media will magnify DM capabilities providing early detection and warning to minimize disaster impact, real time information ongoing and post disaster, and continuous communication to assist support and humanity aid (Seneviratn et al., 2011). This study will present research work in extending existing Disaster Management Metamodel (DMM) with social media and deriving Flood Management Metamodel (FMM) with metamodeling process to visualize the process and activities. The generated output is further modeled with conversion to ontology format to enable conceptualization giving advantage on sharable and reusable information and allowing adding new knowledge regarding the domain.

\section{LITERATURE REVIEW}

In this section the existing works on disaster management knowledge and several works on UML class diagram to OWL ontology mapping is discussed before the actual research work is presented.

\section{Disaster management metamodel (DMM)}

Modeling is a process in designing a conceptual visualization of a domain in real world. It has two core elements which are concept to classify things and relationship that defines connections between entities inside a particular domain (Othman, 2013). This technique empowers visualization since it provides the capability managing difficult complexities (Levendovszky, Rumpe, \& Sprinkle, 2010). This study sets (Othman et al., 2014) as a basis for initial modelling to represent Disaster Management Metamodel (DMM) to elucidate an explicit and scrupulous examination of the DM domain. The DMM has been a comprehensive design covering main aspects of DM which are Mitigation, Preparedness, Response, and Recovery phases.

Existing design have been developed after analyzing and reviewing 37 existing DM models and validated by comparison with other models, Frequency Based Selection (FBS) method to rate the concepts importance, and finally tracing with bushfire case study. However, it is found that this design can be further improved with social media integration under the Situational Awareness concept. Situational Awareness is a condition of having a well-informed knowledge with present situation concerning the person, hence allowing people to predict how the situation will revolve within time and incorporated with the changes of the surrounding environment (Matar, 2017). Enhancing DMM with social media will fall under this category since SA simulates information and communication exchange around the victims and public.

\section{UML to OWL conversion algorithms}

UML is a machine conceptualization used for artefacts specifying, constructing, visualizing, and recording of a software oriented system (Mellor, 2002). In the industry, modelling through UML has been well received and recognized for the purpose of analyzing and designing applications (Eriksson \& Penker, 2000). It allows entities to precisely specifying systems which often reflect in intricate models. The metamodeling process applied in this research work applies UML class diagram to represent the domain model since it is a well-established modeling language generally used by domain experts to encapsulate real world objects in developing object oriented applications (Othman et al., 2014). However, in term of conceptualization it is modeled with OWL ontology language in the needs of applications that require processing the content of information instead of just processing information like class diagram.

\section{Published By:}


Therefore, transformation from UML class diagram to Web Ontology Language (hereafter, OWL) is conducted to map the features while preserving the semantic of the class diagram source. Several works have been done to map UML class diagram to OWL format with one of it is a model called UML2OWL which presents a novel approach by introducing translating algorithm U2OTrans by performing two key level operations ( $\mathrm{Xu}, \mathrm{Ni}, \mathrm{He}, \mathrm{Lin}, \&$ Yan, 2012). The first one is translating UML notations to OWL identifiers and secondly it translates UML elements to OWL axioms. Similar with other works, this model will define one set of mapping rules to translate UML model into OWL ontology. Another approach uses graph transformation and indexing with the Model Driven Architecture (MDA) approach for the automatic construction of functional OWL ontology derived from UML diagram (Belghiat \& Bourahla, 2012). Graphs are practical in describing the fundamental structures of models. With this is mind, this model uses graph transformation to formulate the transformation of visual models. In the graph transformation, graph grammar modelling is used in which applies Chomsky grammar rule. Next model aims taking on consideration of the syntactic and semantic aspects of conceptual data models. In addition it applies information retrieval techniques to define a similarity measure computation. This computation is based on the basic concepts of the conceptual data models (Abdelaziz \& Zakaria, 2014). The key methodology implemented in this work is based on sequence of steps and rules of transformation of conceptual data models in XML Schema. This XML Schema is the core for other analysis rules and transformations to obtain ontology based on the basic concepts that transfers the XML language. The distinct approach used in this technique is it adopts XML as the formal representation format between conceptual data models and ontological models. One of the obstacles in UML to OWL construction is to maintain the semantic of UML class diagram features such as encapsulation, inheritance, associations types i.e. composition and aggregation, integrity constraints, and unique class identifier. To help remediating this issue a work done by exploiting ontology's hierarchy of concepts to represent classes inheritance information within UML is most appropriate as they are focusing on this (Bahaj \& Bakkas, 2013). This approach proposes a determined structure of data type properties to preserve the encapsulation notion, which indicate UML converted attributes visibility levels.

\section{RESEARCH METHODOLOGY}

Model transformation in metamodeling framework is a process of mapping one model to coherent and feasible model. The Meta Object Facility (MOF) framework provides an approach to build different types of metadata in its four metalayers, User Model (level M0), Model (level M1), Metamodel (level M2), and Meta-metamodel (level M3) and applied to instantiate different information models. Model transformation in MOF can be classified in horizontal and vertical dimensions. Horizontal transformation focus on transforming a model into a referred model on the same level of modelling abstraction while vertical transformation execute the transformation of model from one level to a different level of modelling abstraction (Othman \& Beydoun, 2012). For this study the focus will be on horizontal transformation since the DMM will be enhanced on the same level of abstraction with social media capabilities. Vertical transformation will be applied on the step of deriving FMM since it involves instantiation from different level of abstraction.

\section{EXPERIMENTS AND VALIDATION}

An extensive reviews have been conducted in order to extract the most suitable concepts when integrating DMM with social media and designing FMM. Error! Reference source not found. below listed the steps taken.

Step 1: Publications selection from online database based on search strategy.

Step 2: Publications refinement based on inclusion and exclusion criteria.

Step 3: Concepts identification.

Step 4: Concepts validation.

- Step 1.1: Perform Frequency Based Selection.

- Step 1.2: Perform Degree of Confidence.

○ Step 1.3: Design final Metamodel.

Fig. 1 Steps for concepts extraction

From digital databases, many publications are selected based on search strategy formulated with the main keywords disaster and social media. In the literature, social media was sometimes defined in specific such as Twitter and Facebook.

The final search string was ("disaster" OR "disaster management") AND ("social media" OR "Twitter" OR "Facebook"). From here in total 24 publications are relevant to be selected for preliminary review. After performing inclusion and exclusion criteria from Table 1 below to select the best publications only 10 publications are considered the most viable after refinement.

Table 1. Publications refinement inclusion and exclusion criteria

\begin{tabular}{|c|c|}
\hline No. & Inclusion Criteria \\
\hline 1 & $\begin{array}{l}\text { Papers must be focusing on disaster or disaster } \\
\text { management (for DMM). }\end{array}$ \\
\hline 2 & $\begin{array}{l}\text { Papers must be discussing social media usage for disaster } \\
\text { (for DMM). }\end{array}$ \\
\hline 3 & $\begin{array}{l}\text { Papers must be focusing on flood or flood management } \\
\text { (for FMM). }\end{array}$ \\
\hline No. & Exclusion Criteria \\
\hline 1 & Duplicated paper will be excluded. \\
\hline 2 & $\begin{array}{l}\text { Editorials, letters, position papers, and books will all be } \\
\text { excluded. }\end{array}$ \\
\hline 3 & $\begin{array}{l}\text { Papers written in other than English language will be } \\
\text { excluded. }\end{array}$ \\
\hline 4 & Papers without full text will be excluded. \\
\hline 5 & $\begin{array}{l}\text { Papers without full content such as poster, workshop, or } \\
\text { abstract only will be excluded. }\end{array}$ \\
\hline
\end{tabular}




\begin{tabular}{|c|l|}
\hline 6 & $\begin{array}{l}\text { Papers will be excluded if it is not related to disaster } \\
\text { management (for DMM). }\end{array}$ \\
\hline 7 & $\begin{array}{l}\text { Papers will be excluded if the content did not discuss on } \\
\text { social media usage for disaster (for DMM). }\end{array}$ \\
\hline 8 & $\begin{array}{l}\text { Papers will be excluded if it is not related to flood or flood } \\
\text { management (for FMM). }\end{array}$ \\
\hline
\end{tabular}

Similar to DMM above, publications are selected based on search strategy formulated with main keywords which is flood management and in total 12 publications are relevant to be selected as preliminary review. In the literature, flood management tends to be referred as flood mitigation, preparedness, response, or recovery. The final search string was ("flood") OR ("flood management") OR ("flood mitigation") OR ("flood preparedness") OR ("flood response") OR ("flood recovery"). At the end all 12 publications are considered suitable after refinement based on the inclusion and exclusion criteria defined in Table 1 above.

Two techniques have been selected to further validate the correctness of the model proposed. These techniques is called Frequency Based Selection (FBS) and Degree of Confidence (DoC). FBS is a commonly used Feature Selection technique to reduce data variation among researchers. A small number of subset is selected from a number of pertinent features from the initial set. This selection is based on a particular significance determination criteria which tends to provide improved learning accomplishment, lesser computational rate, and improved model interpretability (Tang, Alelyani, \& Liu, 2014). This technique is under on the basis that the ideal model is established with the most coherent features and is frequently defined.

After applying FBS, features or concepts which does not have any correspondence to the classification are eliminated from DMM. FBS technique help to verify the relevance of DMM concepts based on 5 Metamodel quality criteria (Davis \& Bigelow, 2002) which are i) Reasonable representation such as statistical measure of the relative significance of candidate concepts, ii) Provide predictive capability of the Metamodel which is rationally corresponds with initial model throughout the domain, iii) Independent and meaningful variable naming, iv) Highlights all input variables vital in explaining important components of a domain, v) Able to lay out a process flow to the audience in describing how and why the constructed model function as it does.

In this experiment to conduct FBS, several publications are selected based on important perspectives such as role/user, operation, organization, decision, or technology based for flood models. For a selection, a model coverage values $\left(\mathbf{R}_{\text {coverage }}\right)$ are applied as in Table 2 below:

Table 2. Frequency Based Selection (FBS) Coverage.

\begin{tabular}{|c|l|}
\hline$\left(\mathbf{R}_{\text {coverage }}\right)$ & \multicolumn{1}{c|}{ Coverage } \\
\hline 0.3 & Full coverage to entire DM phases. \\
\hline 0.2 & Cover 2 to 3 DM phases in their models. \\
\hline 0.1 & Cover only 1 DM phase. \\
\hline 0.0 & $\begin{array}{l}\text { Did not cover any single DM phase. Will be } \\
\text { eliminated from any further study. }\end{array}$ \\
\hline
\end{tabular}

This process verifies all DMM concepts are validated against other concepts in the models chosen. Based on the Metamodel Transformation Approach this technique obeys vertical model transformation. If required, DMM is enhanced to make sure entire models from the validation sets can be depicted from this model by going through horizontal model transformation approach. By calculating the concept frequency, each concept's importance value within DMM can be computed as the Degree of Confidence (DoC). DoC rate translates the estimated likelihood a DMM concept is applied from a disaster model randomly selected. DoC is obtained by dividing the frequency of how many times a concept used in all selected models with the total number of models. In another words, DoC is referred to list of concepts which used in DMM and defined as below:

DoC $=($ Frequency of Concept $/$ Total Model of Set $) \times 100 \%$

From the DoC it will indicate how much the strength of each concepts is extracted to classify its value accordint to DoC classifications with Very Strong (70 to 100\%), Strong (50-69\%), Moderate (30-49\%), Mild (11-29\%), and Very Mild (0-10\%) (Othman \& Beydoun, 2012). Few relevant concepts have been identified for DMM with social media integration which are Information Dissemination, Volunteered Geographic Information, Evacuation, Sheltering, Animal Management, and Reports. FMM concepts are classified into Structural Mitigation (Structural building measures, Adapted buildings use, Flood barriers, and Flood protection devices) and Non-Structural Mitigation (Land use planning, Financial aid, insurance, Veterinary services, Business continuity management, Re naturalization, Forecasts, Warning systems and education, Borrowing, Adding a cross-border perspective, International aid, Multiple stakeholder strategy decisions, Training, Social science links, Policies, and Production verification).

\section{RESULTS AND DISCUSSION}

Based on the FBS and DoC performed, results of the computation will be discuss below for enhancement of DMM with social media named as DMM v2.0 and initialization of FMM.

\section{DMM v2.0 and FMM}

From the FBS and DoC performed above it can be concluded that Information Dissemination and Volunteered Geographic Information (VGI) are relevant to be included in DMM v2.0 since both are classified as Strong concepts. This is based on the DoC classification table explained above. Information Dissemination is a crucial addition and new approach to the traditional media as a channel for information exchange during a disaster event (Yates \& Paquette, 2011). It enables a medium for collaboration and knowledge sharing (Palen \& Hiltz, 2007).

\section{Blue Eyes Intelligence Engineering}

\& Sciences Publication 
The second concept, Volunteered Geographic Information (VGI) are spatial data produced and shared by public using suitable devices to capture and spread their geographical location on the web. The advantage of VGI is the ability to enhance, update, or complete existing geospatial data sets therefore providing actual data to be gathered such as local information, indicating it is hard to be collected through oldfashioned data capturing processes. In this way it will improve highly comprehensive reports to be generated based on local situations whenever disaster events happen (Gill, Bunker, \& Gill, 2012; Goodchild, 2007). These two concepts will be put under Situational Awareness concept inside Preparedness phase. Nowadays in line with technological advancement, Situational Awareness can be further improved to enable access, analysis, and up to date information concerning to prevailing situations and evolved throughout time (Matar, 2017). Social media are able to cater for these capabilities in information seeking, information verification, and executing queries useful in inaugurating general Situational Awareness (Alsaedi, Burnap, \& Rana, 2015). Therefore social media which falls under situational awareness will be integrated and further classified with Information Dissemination and Volunteered Geographic Information as mentioned above. As mentioned before, FBS is conducted to validate model coverage in a particular perspectives in which any unrelated data set will be unequivocally eliminated. It is discovered there are very low number of Flood Management process publications in particularly Preparedness, Mitigation, Response, \& Recovery thus exhibiting the importance of FMM to be properly documented and modeled. As shown in this evaluation, Flood related concepts is introduced mostly during Mitigation phase. This is primarily due to the reason of lacking in Flood related publications available for reviewing. However, it is perceived that Flood Management Metamodel can be derived from the DMM for its general concepts with few modifications especially on Mitigation process to fit real time flood management processes. It is also discovered from this findings is due to the fact flood Mitigation is the most focus activities during the flood disaster in order to be well prepared rather than remediating during the flood disaster occurrences. From the selected data sets, concepts are identified to be extracted and included into FMM according to its relevancy. Based on the DoC computed, Structural Building Measures and Insurance has scored both $75 \%$ and will be included under Structural and Non-Structural Mitigation respectively.

\section{Mapping UML Class Diagram to OWL Ontology}

Web Ontology Language (OWL) is selected to be constructed from UML due to its efficient reasoners and sufficiently expressive for complex classes of ontologies (Harmelen \& Antoniou Frank van, 1993). An algorithm is adapted to address the purpose of this research (Bahaj \& Bakkas, 2013). As described descriptively before, this work comprises of an algorithm to perform conversion from UML class diagram to ontology while preserving the semantic of the UML's features such as inheritance, encapsulation, types of associations, constraints of integrity, and class identifier. Fig below is describes the mapping steps taken within the conversion algorithm while Fig depicts the algorithm mapping system architecture.

\begin{tabular}{|c|c|}
\hline Step 1: & Map Meta Model into Conversion Template. \\
$\circ$ & Step 1.1: Identification of class name. \\
$\bigcirc$ & Step 1.2: Identification of class parent. \\
$\circ$ & Step 1.3: Classification of attributes list. \\
$\circ$ & Step 1.4: Classification of relationship list. \\
Step 2: & Execute the algorithm. \\
$\circ$ & Step 2.1: Create and initialize Object. \\
$\bigcirc$ & Step 2.2: Map Meta model classes. \\
$\circ$ & Step 2.3: Map attribute list. \\
$\circ$ & Step 2.5: Map relationship list. \\
$\circ$ & Step 2.6: Generate ontologv generation as output. \\
\hline
\end{tabular}

Fig. 2 Mapping steps for UML to OWL conversion

Taking the DMM v2.0 Mitigation metamodel source as an example, the first main step is to map into a conversion template (.txt file). Class name such as Insurance is identified with the parent class if available, or not initialized with Object. The attribute for this class is also listed with the relationship among other classes. This mapping can be represented in the template such as (Insurance, Object, \{(Insurance_ID, private, 1 , string, false). The next main step will parse the input template and mapped all the classes and axioms accordingly thus producing OWL ontology file at the end. OWL object is created in which for this case is the owl:Thing class. All metamodel classes including the parent, attributes, and relationships will be mapped accordingly with the mapping rules (Bahaj \& Bakkas, 2013). OWL files is an XML-based syntax, which makes this syntax is human readable and easy to understand (Harmelen \& Antoniou Frank van, 1993). This algorithm is written with Java programming language and Apache Jena Ontology Application Program Interface (API). Jena is a Java based framework which consists of large Java libraries specially used for Semantic Web applications development (Jauro, 2014).

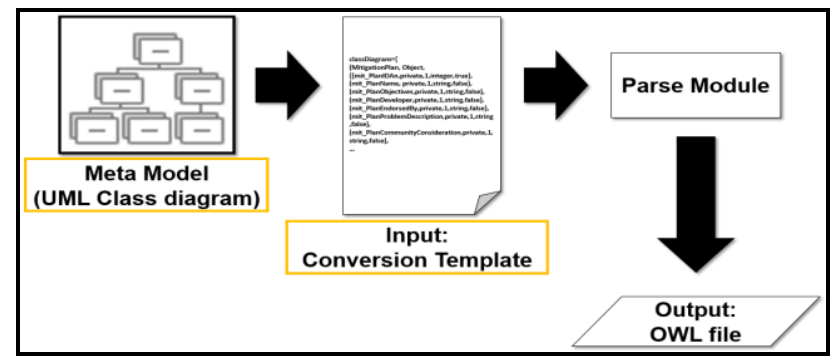

Fig. 3 UML to OWL System Architecture (Bahaj \& Bakkas, 2013).

As an example utilizing Mitigation metamodel source and using the ontology output file generated, it was loaded into Protégé, an open-source ontology editor and framework for Semantic Web development.

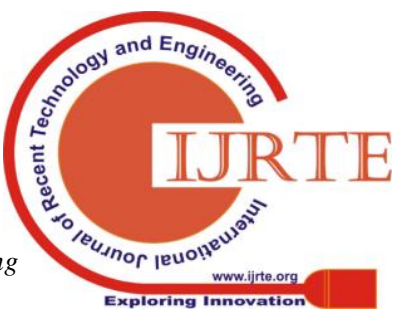




\section{Metamodeling to Ontology Conversion with Social Media Influence in Flood Management Domain}

Protégé functionality includes rule-based reasoners (e.g. ELK, FaCT++, HermiT, and Pellet) capable to deliver semantic varification of the OWL language for a set of ontology data provided (Dickinson, 2004). After performing the validation, the ontology source able to run and conceptualize explicitly as shown in Fig below by OntoGraf function. From here all relationships between one concepts to another can be referred closely.

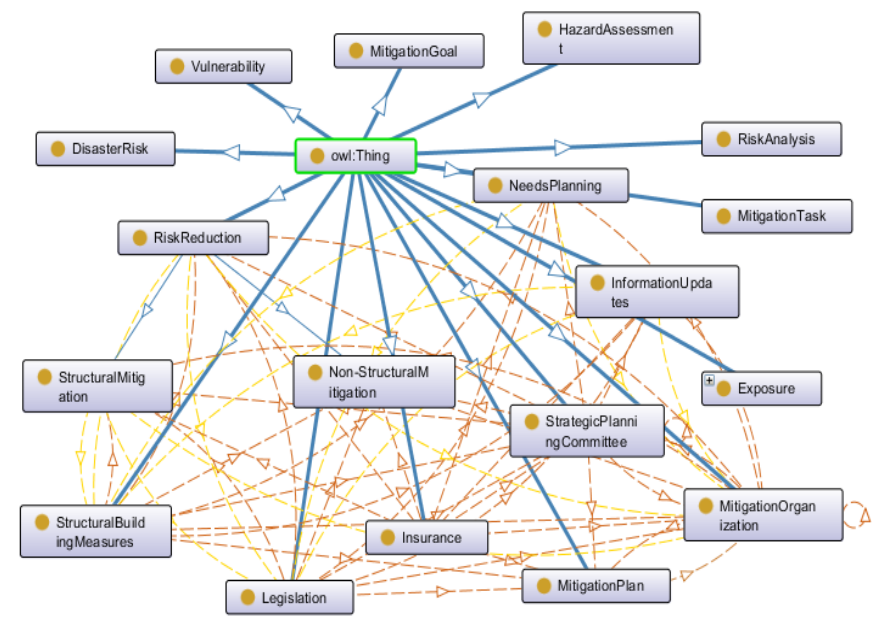

\section{Fig. 4 Generated Mitigation Phase ontology visualized with OntoGraf function in Protégé.}

\section{CONCLUSION}

This study extends the existing DMM design with social media capabilities by introducing two new concepts which are Information Dissemination and Volunteered Geographic Information associated with Situational Awareness within Preparedness phase. Secondly, based on the DMM a new disaster centric metamodel is generated specifically for flood called Flood Management Metamodel. Based on the analysis completed, two new concepts are also introduced under Structural and Non-Structural Mitigation which are Structural Building Measures and Insurance respectively. From the metamodel introduced, this research work continues to adapt ontology mapping algorithm to facilitate UML class diagram to OWL ontology conversion. The result shows the metamodel able to conceptualize effectively while preserving the semantic relationships. Future work includes enhancing the algorithm to provide automated conversion without using a class template as input.

\section{REFERENCES}

1. Abdelaziz, T., \& Zakaria, E. (2014). Construction of a domain ontology from the conceptual data models.

2. Alsaedi, N., Burnap, P., \& Rana, O. (2015). Identifying Disruptive Events from Social Media to Enhance Situational Awareness. Proceedings of the 2015 IEEE/ACM International Conference on Advances in Social Networks Analysis and Mining 2015 - ASONAM '15, (November), 934941. https://doi.org/10.1145/2808797.2808879

3. Atmanand. (2003). Insurance and disaster management: The Indian context. Disaster Prevention and Management: An International Journal, 12(4), 286-304. https://doi.org/10.1108/09653560310493105
4. Bahaj, M., \& Bakkas, J. (2013). Automatic Conversion Method of Class Diagrams to Ontologies Maintaining Their Semantic Features. International Journal of Soft Computing and Engineering (IJSCE), (6), 65-69.

5. Belghiat, A., \& Bourahla, M. (2012). An Approach based AToM3 for the Generation of OWL Ontologies from UML Diagrams, 41(3), 41-48.

6. Bosher, L., Andrew Dainty, Patricia Carrillo, \& Glass, J. (2007). Built-in resilience to disasters : a pre-emptive approach. Engineering, Construction and Architectural Management, 14(5), 434-446.

7. Calton, P., \& Masaru, K. (2013). Big Data and Disaster Management A Report from the JST / NSF Joint Workshop. Technical Report No. GITCERCS-13-09; Georgia Institute of Technology, CERCS., (May), 28. Retrieved from https://grait-dm.gatech.edu/wpcontent/uploads/2014/03/BigDataAndDisaster-v34.pdf

8. Cameron, M. A., Power, R., Robinson, B., \& Yin, J. (2012). Emergency situation awareness from twitter for crisis management. Proceedings of the 21st International Conference Companion on World Wide Web WWW '12 Companion, (September 2014), 695. https://doi.org/10.1145/2187980.2188183

9. Davis, P. K., \& Bigelow, J. H. (2002). Motivated Metamodels. Proceedings of The 2002 PerMIS Workshop, (February).

10. Deshmukh, Ravindra, Lewlyn L. R. Rodrigues, G. R. K. (2008). Earthquake Risk and Knowledge Management. Journal of Knowledge Management Practice, 9(3), 376-390. https://doi.org/10.3846/ijspm.2010.28

11. Dickinson, I. (2004). Implementation experience with the DIG 1.1 specification. Filton Road, Stoke Gifford, Bristol, UK, BS34 8QZ: Semantic and Adaptive Systems Dept, HP Labs Bristol.

12. Eriksson, H., \& Penker, M. (2000). Business Modeling With UML. Business Patterns at Work, 12. https://doi.org/978-0471295518

13. Gill, A., Bunker, D., \& Gill, A. Q. (2012). Association for Information Systems AIS Electronic Library (AISeL) Crowd Sourcing Challenges Assessment Index for Disaster Management Crowd Sourcing Challenges Assessment Index for Disaster Management, (1). Retrieved from http://aisel.aisnet.org/amcis2012\%0Ahttp://aisel.aisnet.org/amcis2012/pro ceedings/EGovernment/21

14. Goodchild, M. F. (2007). CITIZENS AS SENSORS: THE WORLD OF VOLUNTEERED GEOGRAPHY 1 Michael F. Goodchild. Geojournal, 69(4), 1-15.

15. Harmelen, G., \& Antoniou Frank van. (1993). Web Ontology Language: OWL. https://doi.org/10.1145/1295289.1295290

16. Jauro, F. (2014). Design And Implementation Of Falcon-AO++: An Improved Ontology Alignment System. Ahmadu Bello University, Zaria. Retrieved from http://kubanni.abu.edu.ng:8080/jspui/handle/123456789/5623

17. Jha, A. K., Barenstein, J. D., Phelps, P. M., Pittet, D., \& Sena, S. (2010). Safer Homes, Stronger Communities. Construction. https://doi.org/10.1596/978-0-8213-8045-1

18. Levendovszky, T., Rumpe, B., \& Sprinkle, J. (2010). Model-Based Engineering of Embedded Real-Time Systems (Vol. 6100). https://doi.org/10.1007/978-3-642-16277-0

19. Lloyd-Jones, T., Kalra, R., Mulyawan, B., \& Theis, M. (2009). The Built Environment Professions in Disaster Risk Reduction and Response. Max Lock Centre at the University of Westminster. https://doi.org/10.1103/PhysRevLett.90.138102

20. Matar, A. (2017). Enhancing Situational Awareness and Communication During Flood Crisis Events Using Social Media Framework : The Case of Bosnia and Herzegovina, (May), 1-320.

21. Mellor, S. J. (2002). Executable UML: a foundation for model-driven architecture. Addison-Wesley Professional, 1-9.

Published By: 
22. Moe, T. L., Gehbauer, F., Senitz, S., \& Mueller, M. (2007). Balanced scorecard for natural disaster management projects. Disaster Prevention and Management: An International Journal, 16(5), 785-806. https://doi.org/10.1108/09653560710837073

23. Munich, R. (2013). Münchener Rückversicherungs-Gesellschaft. Geo Risiko Forschung, NatCatSERVICE.

24. Othman, S. H. (2013). Supporting Domain Ontology through a Metamodel: A Disaster Management Case Study. In R. M. Colomb (Ed.), Ontology-Based Applications for Enterprise Systems and Knowledge Management (1st ed., pp. 191-209). USA: IGI Global. https://doi.org/10.4018/978-1-4666-1993-7.ch011

25. Othman, S. H., \& Beydoun, G. (2012). Evaluating disaster management knowledge model by using a frequency-based selection technique. Lecture Notes in Computer Science, 21-27.

26. Othman, S. H., Beydoun, G., \& Sugumaran, V. (2014). Development and validation of a Disaster Management Metamodel (DMM). Information Processing and Management, 50(2), 235-271. https://doi.org/10.1016/j.ipm.2013.11.001

27. Palen, L., \& Hiltz, S. R. (2007). Online Forums Supporting Grassroots Participation in Emergency Prepardness and Response, (June 2014). https://doi.org/10.1145/1226736.1226766

28. Poblet, M., Garcia-Cuesta, E., Casanovas, P., García-cuesta, E., \& Casanovas, P. (2014). Crowdsourcing Tools for Disaster Management: A Review of Platforms and Methods. AI Approaches to the Complexity of Legal Systems, 8929(Ccc), 261-274. https://doi.org/10.5751/ES-05263170431

29. Porto de Albuquerque, J., Horita, F. E. A., Degrossi, L. C., Rocha, R. dos S., Camargo de Andrade, S., Restrepo-Estrada, C., \& Leyh, W. (2017). Leveraging Volunteered Geographic Information to Improve Disaster Resilience, (March), 158-184. https://doi.org/10.4018/978-1-5225-24465.ch009

30. Rahmat, N. A., Hagishima, A., Ikegaya, N., \& Tanimoto, J. (2016). An experimental study on aerodynamic interaction between a boundary layer generated by a smooth and rough wall and a wake behind a spire. Engineering Sciences Reports, 37(2), 19-26.

31. Seneviratn, K., Chaminda Pathirage, Dilanthi Amaratunga, \& Richard Haigh. (2011). Disaster knowledge factors: benefits and challenges. https://doi.org/10.1227/01.neu.0000467148.96632.84

32. Slamet, C., Rahman, A., Sutedi, A., Darmalaksana, W., Ramdhani, M. A., \& Maylawati, D. S. (2018). Social Media-Based Identifier for Natural Disaster. IOP Conference Series: Materials Science and Engineering, 288(1). https://doi.org/10.1088/1757-899X/288/1/012039

33. Tang, J., Alelyani, S., \& Liu, H. (2014). Feature selection for classification: A review. Data Classification: Algorithms and Applications, 37-64. https://doi.org/10.1201/b17320

34. Underwood, S. (2010). Improving disaster management. Communications of the ACM, 53(2), 18. https://doi.org/10.1145/1646353.1646362

35. Wallemacq, P., Guha-Sapir, D., McClean, D., CRED, \& UNISDR. (2015). The Human Cost of Weather Related Disasters - 1995 - 2015.

36. Xu, Z., Ni, Y., He, W., Lin, L., \& Yan, Q. (2012). Automatic extraction of OWL ontologies from UML class diagrams: A semantics-preserving approach. World Wide Web, 15(5-6), 517-545. https://doi.org/10.1007/s11280-011-0147-z

37. Yates, D., \& Paquette, S. (2011). A case study of the 2010 Haitian earthquake. International Journal of Information Management, 31(1), 613. https://doi.org/10.1016/j.ijinfomgt.2010.10.001 